\title{
« Les coulisses de la création »1
}

\author{
Anita Lavernhe-Grosset \\ Université du Littoral Côte d'Opale
}

Tous les arts vivent de paroles. Toute œuvre exige qu'on lui réponde, et une "littérature", écrite ou non, immédiate ou méditée, est indivisible de ce qui pousse l'homme à produire, et des productions qui sont les effets de ce bizarre instinct. (Valéry, 2016e, p. 1329)

Dans ses Pièces sur l'art, Paul Valéry donne ainsi une définition possible du commentaire critique comme forme seconde et

1 Valéry, 2016e, p. 1330. 
nécessaire d'une dynamique de dialogue inscrite dans l'œuvre et participant d'elle. Le mot "littérature » qualifie la critique comme expérience de l'effet esthétique selon un système de réciprocité interne à l'œuvre qui articule à la pulsion de l'écriture, son attente essentielle, les commentaires, "productions qui sont les effets de ce bizarre instinct». Le critique, en ce sens, accomplit l'œuvre dans une langue commune et la tire du néant qui la menace; non seulement il l'actualise mais cet échange donne sens à l'insensé de l'écriture et la soustrait à sa singulière solitude. Davantage, « le critique ne doit pas être un lecteur mais le témoin d'un lecteur, celui qui le regarde lire et être mû » (2016f, p. 265), écrit Paul Valéry. Monsieur Teste, figure emblématique de l'écrivain, incarne dès lors le critique comme testis, comme témoin. Car pour Valéry, «l'opération critique capitale est la détermination du lecteur. La critique regarde trop vers l'auteur.» (2016f, p. 265) Ainsi l'œuvre advient en chaque lecture, autonome de son auteur et virtuellement actuelle, tout en possibles, à partir des effets qu'elle produit et qu'il échoit à l'expérience critique, comme esthétique, d'exhausser. La Nouvelle Critique des années 1960 en tirera les conséquences.

Entendre l'exigence de dialogue des œuvres est, pour Valéry, interroger le travail d'écriture, explorer le faire, ce qu'après lui, on désignera comme la fabrique du texte :

Ce qui m'intéresse - quand il y a lieu - ce n'est pas l'œuvre - ce n'est pas l'auteur-c'est ce qui fait l'œuvre.

Toute œuvre est l'œuvre de bien d'autres choses qu'un "auteur".

Je connais la littérature pour l'avoir interrogée à ma guise. (Et seulement ainsi). (2016g, p. 477) 
Car c'est la genèse de l'œuvre que l'écrivain, admirateur de Poe, veut comprendre, "par-dessus l'épaule de l'auteur» (2016g, p. 475), là où « la littérature joue quelquefois, dans les coulisses de la création, un rôle assez remarquable » (2016e, p. 1330).

L'originalité inaugurale de cette pensée, nous avons voulu l'éprouver. Comment va réagir le témoin «[des] effets proprement littéraires du langage », qui affirme, en 1937, que "la Littérature est, et ne peut être autre chose qu'une sorte d'extension et d'application de certaines propriétés du Langage » (2016j, p. 947-948)? Qu'en sera-t-il de ces «propriétés profondes », qu'il nomme « sensibilité formelle » (2016f, p. 259)? Nous tenterons d'y répondre par l'étude de Variation sur une "pensée », article fameux et commenté de Valéry sur Pascal, parce qu'il témoigne, croyons-nous, chez ce précurseur de la Nouvelle Critique, d'une impossibilité heuristique.

\section{Contextes}

Le 8 mars 1902, Paul Léautaud note dans son .Journal littéraire :

P. V. causeur merveilleux. Tout le monde fait cercle au Mercure, le mardi, pour l'entendre. Des vues extrêmement originales. Une façon extra-intellectuelle de voir et définir les choses de la littérature dans toutes leurs combinaisons, ce mot, entendu presque dans un sens de laboratoire, ou de clinique, ou de calculs algébriques, toutes les parties soigneusement préméditées, l'élément artistique gardant toutefois son rôle, il dirait presque : son jeu.

[...]

Autre côté : très impressionné, séduit, par l'histoire des Pensées de Pascal, écrites, griffonnées sur des morceaux de papier, jetés pêle-mêle dans un sac. Je sens cela chez lui sans qu'il m'en ait 
dit plus que quelques mots. Un certain côté de son esprit aussi... Nous ne parlons guère de Pascal que pour nous gausser de la sottise de ses annotateurs. (2005, p. 27)

Léautaud conçoit finement l'écrivain expérimentateur d'une littérature, où la pensée fait jeu avec la matière verbale en un perpétuel exercice de poïétique. Ajoutons que la pratique fragmentaire des Cahiers de Valéry n'est pas sans affinité avec «l'histoire des Pensées de Pascal », comme l'écrit Paul Léautaud, ni les Carnets de Léonard, qui lui inspirent «l'impression extraordinaire d'un ensemble hallucinant d'étincelles arrachées par les coups les plus divers à quelque fantastique fabrication » (2016h, p. 825). Cette observation passionnée de la création en acte est l'étrangeté de Valéry. On sait qu'après La Crise de Gênes de 1892, le créateur suspendra vingt ans toute publication, pour explorer le système de sa conscience par des exercices matinaux de pensée, jetés en notes sur des cahiers. Chaque aube, jusqu'à sa mort, l'écrivain répétera l'étonnant rituel. Il s'en explique dans une lettre de 1929 à Georges Duhamel :

Je me livrais, - depuis 1892, - à des pensées et à des problèmes toujours plus éloignés de la poésie, et même de toute littérature praticable. Plus j'allais, plus j'étais sûr, sans même y songer, de ne revenir jamais à l'exercice des lettres. J'accumulais seulement des notes ou idées, mais si diverses, et si libres de toute intention de les utiliser, que la seule pensée de les reprendre et d'en faire quelque ouvrage, me paraissait absurde. (1997, p. 179)

Cette tension essentielle montre un Valéry bifrons. Face mondaine œuvrant de commande; ainsi des études réunies dans Variété et de nombreux écrits de circonstance. Face noble, appliquée à la poésie pure et à l'écriture des grands dialogues, dédiée surtout à l'extraordinaire recherche des Cahiers. 
Telle apparaît Variation sur une «Pensée ${ }^{2} »$. Commande, c'est une œuvre de circonstance, «besogne courante », commente une lettre à Pierre Féline de 1923 (1957, p. 1739) : "rien de digne d'être communiqué à d'autres qu'à 40.000 lecteurs de la R. H. » Mais comment ne pas voir la présence insistante de Pascal dans les différentes proses de Valéry, partagé entre admiration et exaspération? La « besogne » paraît prétexte à un désir confié, dès 1917, à son ami Pierre Louÿs (1952, p. 121) : «J'ai indiqué à mon cerveau futur les principes directeurs d'un éreintement sauvage des Pensées de Pascal (au bénéfice du Traité de l'équilibre des liqueurs). " Ce meurtre symbolique de l'écrivain janséniste pour mieux exalter en lui le savant invite à concevoir sous la fascination de Valéry, éparse dans toutes ses marges, la figure de Pascal comme la face nocturne de Léonard l'Apollinien :

Noir. Les "pessimistes de la plume" : ils cherchent un "beau noir", dirait un peintre.

Pascal a de "beaux noirs" et les a cherchés, et je vois trop qu'il les a trop bien trouvés. (2016d, p. 123)

Lors du tricentenaire de Blaise Pascal, la Revue Hebdomadaire $^{3}$ livra, pour son numéro du 14 juillet 1923, des articles de Maurice Barrès, Henri Brémond, Jacques Maritain, François Mauriac, Paul Desjardins, Julien Benda, Charles Du Bos, Jacques Chevalier, etc. Paul Valéry écrivit Variation sur une "Pensée ». L'article fit sensation. Repris dans Variété en 1924, il est ensuite enrichi d'annotations en italiques envisageant le texte. C'est dans cette version définitive, annotée par l'auteur, qu'il figure en 1937 en l'édition des OEuvres, réalisant ainsi, dans

2 La majuscule apparaît dans l'article de 1919 et disparaît dans l'ultime version de 1937.

${ }^{3}$ Revue Hebdomadaire, $32^{\mathrm{e}}$ année, tome VII, $\mathrm{n}^{\circ} 28$. 
l'esprit des Cahiers, un exercice de Self-Variance (1990a, p. 408) :

Il y a changement.

Pour que ce changement ait lieu...... $\rightarrow$ Il a toujours lieu, il est fondamental.

LOI DE LA VARIATION DES ÉTATS DE CONNAISSANCE. COURBE DE LA SELF-VARIANCE.

Déployant le commentaire, ces « NOTES DE L'AUTEUR ${ }^{4}$ » ne forment pas seulement une variation de la variation mais encore sa défense et illustration. Car

certains parlèrent de scandale. Même quelques hommes des plus éminents de ce temps-ci se sont trouvés blessés comme dans leur personne du résultat d'un examen que chacun peut faire soi-même. - Faut-il donc y revenir? - Qu'ai-je donc fait? (2016i, p. 796)

La «Pensée » de Pascal, "Le silence éternel de ces espaces infinis m'effraie », est l'objet que se donne Valéry, qui précise dans les "Notes», qu'elle "a servi de thème à la présente "Variation", par laquelle on n'entendit rien proposer qui ne se déduisît presque inévitablement de ce texte si beau et si bref » (p. 792). Cette remarque met l'accent sur des constantes valéryennes: pour la pensée abstraite, la contrainte de la rationalité dans l'analyse et l'idée que l'œuvre ne peut se comprendre qu'en elle-même, sans extériorité, du seul point de vue de son langage par un refus répété de l'histoire littéraire.

4 NOTES DE L'AUTEUR, en italiques sur les pages paires du texte. Nous présenterons celles-ci sous cette forme : P. 788, 790, etc. Les pages impaires, en lettres normales, constituent le TEXTE inaugural de Valéry. 


\section{Variation}

Bien nommée, cette étude littéraire fait une lecture déviante et affranchie :

Le développement de notre "Variation" nous a conduit naturellement à opposer l'idée que Pascal nous donne de soimême, (ou bien, celle que nous présente de lui la tradition), à l'idée que nous pouvons nous en faire, par nous-mêmes. (p. 796)

Ainsi, ce qui varie dépend de l'orientation de deux idées, l'une tournée vers l'auteur, l'autre, vers le lecteur par effet de lecture. Rappelons à ce propos les $R h u m b s^{5}$ comme métaphore de l'écart, notion que reprendra Adorno (1999) comme titre d'un article majeur sur Valéry. En épigraphe ironique à Variation sur une "pensée », la citation LE SILENCE ETERNEL.... (p. 789) suivie de quatre points de suspension : le «Robinson intellectuel » des Cahiers (1990b, p. 422) ou encore de L'Idée fixe (2016c, p. 118), suspend le topos dans le vide, tranche à vif ce qui ne pense plus. La variation pointe l'invariant, faisant, comme le note Valéry, "d'une des paroles les plus fameuses » (2016i, p. 788), le lieu commun de la littérature pascalienne. Cet exercice de Self-variance va rassembler, pour une expérience de pensée, le corps, l'esprit et le monde, que les Cahiers systématisent sous les trois lettres $\mathrm{CEM}^{6}$ pour substituer à l'expérience pascalienne de l'effroi la contemplation d'autres regards, dans des conditions de pensée et de culture différentes. Ainsi, renversant l'argument de Pascal, « une certaine variation

\footnotetext{
5 Titre choisi par Valéry pour rassembler des fragments de Tel Quel II.

6 "Mon idée est de considérer "mon" Corps - "mon" Monde - "mon" esprit, comme trois variables principales entre lesquelles la vie sensitivo-consciente et agissante (à partir d'un moi) est relation. » Cahiers, XXV, fac-similé original, Paris, CNRS, 1957, p. 178.
} 
(déjà sensible)» (p. 809), écrit Valéry, va apaiser notre intelligence du monde, en «[rapprochant] ce qui était si stupéfiant et si émouvant de ce qui est familier à nos sens, accessible à notre action, et qui se conforme d'assez près à nos raisonnements » (p. 807).

\section{L'intertextualité et le mimétisme critique}

S'engage une expérience critique, qui prend d'abord la forme d'un intertexte poétique car la Pensée de Pascal, dit Valéry,

[...] est un Poème, et point du tout une Pensée.

Car Éternel et Infini sont des symboles de non-pensée ${ }^{7}$. (p. 788)

Or, si paradoxalement, " une forme est féconde en idées » (2016a, p.550), la sensibilité formelle qu'elle met en œuvre poétiquement défie ici Pascal dans le dialogue philosophique, forme fétiche de l'écrivain. C'est de la musicalité composée de la variation valéryenne et de sa résonance poétique que la confrontation des représentations surgira : l'effroi de l'homme pascalien dans le silence de l'univers infini contredit par la tranquillité de l'âme charmée de la cosmologie antique. Dans la nuit transfigurée, la conscience écoute la musique des sphères. Valéry offre ainsi une sorte de paragone, où la lecture sensible d'un dialogue exerce une fonction contradictoire en regard de la citation, «LE SILENCE ETERNEL....», lacune épigraphe, dans l'éclat ironique des capitales :

\footnotetext{
7 Voir le commentaire de la même Pensée dans Claudel (1963 [1993]), p. 7374 : «Dissyllabe net et ouvert sur un blanc faisant équilibre à lui seul à cette grande phrase légère et spacieuse composée de quatre anapestes. Remarquez en soutien le choc sourd des deux nasales en et in. Aussi cette espèce de déhiscence sidérale entre espaces et infinis. »
} 
Quels sons doux et puissants, demande Eustathe à Pythagore, et quelles harmonies d'une étrange pureté il me semble d'entendre dans la substance de la nuit qui nous entoure? (p. 789)

Ainsi commence la variation, par cette transcription du Songe de Scipion de La République (livre VI) de Cicéron, à partir d'une réécriture poétique du Commentaire qu'en fait Macrobe, sur l'harmonie des astres et la musique des sphères. Appliquant à la prose des «variations sur le classique » (2016f, p. 271), l'écrivain crée "la distance entre la "pensée" initiale et "l'expression" finale [...] la plus grande possible » (2016f, p. 272) et devient «traducteur» (p. 272). «Une belle infidèle » (p. 272) donne à lire, en abîme, la rature et la réécriture du palimpseste, découvert en 1819 par le philologue et cardinal Angelo Mai, qui fit apparaître, sous des commentaires de Saint-Augustin sur les Psaumes, "Le Songe de Scipion » du De Republica de Cicéron (Bayet, 1965, p. 133, n. 2). Valéry opère ainsi un renversement pour le moins abrasif par une sorte de déconstruction du paradigme pascalien, en un jeu qui part de la rature de SaintAugustin pour composer la fusion d'un texte et de son commentaire et, par l'anachronisme des interlocuteurs, disposer le temps en un espace purement littéraire. L'article de 1923 peut donc être lu comme suite et variation sur une "pensée », variété et modulation moderne d'une expérience littéraire et philosophique de l'univers depuis l'Antiquité. Le commentaire critique de Paul Valéry se constitue ici comme la variante « d'une Histoire approfondie de la Littérature [...] une Histoire de l'esprit en tant qu'il produit ou consomme de la "littérature"» (2016j, p. 946).

Car le travail critique de Valéry inverse la thématique pascalienne par la convenance d'une forme poétique, «la 
littérature réduite à l'essentiel de son principe actif » (2016f, p. 256). C'est ainsi que la composition musicale des sensations naît des effets sensibles d'assonances et d'allitérations filées. comme l'affirme le poète dans Tel Quel I :

Et le "magnétisme" de la voix doit se transposer dans l'alliance mystérieuse et extra-juste des idées ou des mots.

La continuité du beau son est essentielle. (p. 258)

Parce qu'elle est substantielle, la nuit acquiert une densité enveloppante et, dans l'harmonie de ses échos, elle est résonance (2016e, p. 1340), chiasme sonore. Nous ne pouvons qu'en suggérer le charme. Le ciel résonne. Eustathe entend la dissemblance essentielle du son et interroge Pythagore sur le ravissement musical de son âme. Sa conscience, dans l'univers, ne s'égare pas mais se recentre. Au lieu de l'effroi d'un moi fragmenté et perdu dans le silence inhumain de l'espace, s'éprouve le bien-être d'une conscience contemplative. À l'extrême de l'ouïe, la félicité rayonne en des correspondances perceptives, intellectuelles, dans le sentiment euphorique de la «surprise» (p. 789); correspondances morales encore car l'âme «se tend, pareille à l'espérance » (p.789), "jusqu'aux limites de mon sens» (p. 789), dit-elle. L'émotion spirituelle l'étreint: "Quel est donc le mystérieux instrument de ces délices? » (p. 789)

Le dialogue entre Pythagore et Eustathe contredit le discours pascalien et l'expérience contemplative du disciple parachève la connaissance du savant antique qui donne sens à l'extase. Se superposent ainsi les expériences par contraste et modulation. "L'ordre du monde pur, dit le philosophe, enchante tes oreilles. L'intelligence, la justice, l'amour, et les autres perfections qui règnent dans la partie sublime de 
l'univers, se font sensibles. » (p. 791) Pythagore enseigne ici une conception divine du Cosmos, ordonné, rationnel, et devenu « sensible au cœur» (Pascal, 2009, p. 467, s. 680) : «Il n'y a point de silence dans l'univers. » (p. 789) Valéry le fait encore entendre en invoquant la tradition judéo-chrétienne des Psaumes de David: "Quant aux Juifs, ils ne parlent des cieux qu'ils n'en célèbrent l'éloquence » (p. 791), écrit-il, dans l'éclat des noms et des verbes. La voix de Pascal dissone, et Valéry fait retentir sa dissonance par sa seule citation, coupée en son centre et resserrée sur un désert, privé d'un Robinson : " "en regardant tout l'univers muet, il entre en effroi, dit-il, comme un homme qu'on aurait porté endormi dans une île déserte et effroyable..." » (p. 793)

La méditation finale ajoutera aux précédentes la contemplation valéryenne du ciel nocturne, en une nouvelle variation qui fait apparaître l'homme sans Dieu et sans espérance de Pascal, et qualifier celui-ci, en une dépréciation cinglante, «d'étrange chrétien» (p. 793). Par le choix d'une écriture palimpseste, Valéry dévoile la transformation dans le temps des représentations humaines, l'angoisse pascalienne offrant un contraste inhumain aux réponses accordées du cœur qui s'invente un Dieu et de l'esprit scientifique qui crée le sens.

\section{La construction de l'auteur}

À la démonstration poétique va s'ajouter un travail sur la figure de Pascal et la notion d'autorité, car pour l'auteur de L'Introduction à la méthode de Léonard de Vinci et de Monsieur 
Teste, «de personis non curandum ${ }^{8}$ » (2016b, p. 1080). Sur la scène de l'imaginaire, Valéry traque le mythe, les idées fixes, les idoles (1990a, p. 544) et autres superstitions littéraires (2016g, p. 569) : élaboré par l'histoire, amplifié par le romantisme, Pascal est le «personnage le plus noir, le plus pur de la Légende Intellectuelle » (2016i, p. 796), "l'Hamlet français et janséniste » (p. 799), « une sorte de Héros de la dépréciation totale et amère » (p. 796). Or, accusant l'intelligence de l'auteur des Pensées, le critique, par système, va substituer au nom de Pascal le pronom " elle», féminin insolite, qui soumet toute la lecture à une variation heuristique. Ainsi, dans l'écart, œuvre Teste, ce "témoin principal qui regarde tous [les] fantoches s'agiter ${ }^{9}$ » (1997, p. 22) en lui. Ce déplacement participe de ce que Poésie et Pensée abstraite nomme encore "le nettoyage de la situation verbale » (2016j, p. 821). L'expérience critique consiste donc en ce travail d'hygiène mentale - par analogie «à la mode des chirurgiens» (p. 821), aseptisant le champ opératoire pour décrasser, en quelque sorte, "l'impureté10" de l'esprit et du langage en une déconstruction des concepts, comme l'expriment gaiement les deux protagonistes de L'Idée fixe: « vous avez la bosse de l'étonnement, mon cher. - Oui... Je dois avoir une boîte de résistances quelque part dans le cerveau... », dit le médecin au narrateur, et celui-ci conclut ce moment: Vous ferraillez pour et contre les idées, comme un beau diable, et exterminez le cacatoès... » (2016c, p. 231-239)

\footnotetext{
8 Traduction de l'éditeur. «Il ne faut pas se soucier des personnalités / des caractères. »

${ }^{9}$ La phrase s'achève par deux lignes de points de suspension.

10 Notion récurrente (en forme de polyptote) dans la Variation. Tel Quel II : "Apologétique est impureté », p. 682.
} 


\section{La folie de Pascal et le moi haïssable}

C'est pourquoi, considérant la sensibilité intellectuelle du lecteur et l'exigence d'une Éthique de l'esprit (2016i, p. 804), Valéry dénonce en Pascal l'Apologiste (p. 804) et l'accuse d'impureté dans les moyens et dans les intentions. Il procède à une paraphrase critique des Pensées, qui décèle une pensée excessive, l'ubris de l'intelligence créatrice: "Effroi, effrayé, effroyable; silence éternel; univers muet, c'est ainsi que parle de ce qui l'entoure l'une des plus fortes intelligences qui aient paru.» (p. 793) La liste fait violence. L'effet de copia, le martèlement délibéré des mots et la contradiction finale forcent l'esprit, non seulement à la déréliction mais au scandale d'un univers insensé, sans ordre et sans Logos. Valéry attaque la perversion de l'intelligence dans le pouvoir de connaître le monde. Sujet de verbes réfléchis, la conscience sensible, inscrite dans le mouvement de l'écriture, construit une pensée de l'inhumain :

Elle se ressent, elle se peint, et se lamente, comme une bête traquée; mais de plus, qui se traque elle-même, et qui excite les grandes ressources qui sont en elle, les puissances de sa logique, les vertus admirables de son langage, à corrompre tout ce qui est visible et qui n'est point désolant. (p. 793)

L'insistance des occurrences et la contradiction de l'intelligence représentée comme «bête traquée / qui se traque elle-même » marquent la détermination critique de Valéry à dévoiler la duplicité de l'esprit créateur dans ses variations paradoxales, qu'on retrouve dans l'association antagonique: «ce qui est visible et qui n'est point désolant». Pour rendre l'outrance sensible, l'auteur va concentrer l'esprit de l'œuvre, 
comme pouvoir subordonné à un vouloir ${ }^{11}$ (2016a, p. 572) par le mimétisme critique d'une forme du désastre. Le texte valéryen compose une interprétation experte, en un pastiche miniature, qui parodie, sous la pression du sujet féminin, la manigance de l'intelligence, la volonté paranoïaque du savant dévoyé, subvertissant sa raison à échafauder des délires :

Elle se veut fragile et entièrement menacée, et de toutes parts environnée de périls et de solitude, et de toutes les causes de terreur et de désespoir. Elle ne peut souffrir qu'elle soit tombée dans les filets du temps, du nombre et des dimensions, et qu'elle se soit prise au piège du système du monde. Il n'est pas de chose créée qui ne la rappelle à son affreuse condition, et les unes la blessent, les autres la trompent, toutes l'épouvantent, tellement que la contemplation ne manque jamais de la faire hurler à la mort. Elle me fait songer invinciblement à cet aboi insupportable qu'adressent les chiens à la lune; mais ce désespéré, qui est capable de la théorie de la lune, pousserait son gémissement tout aussi bien contre ses calculs. (p. 793 795)

Toute la suite du texte développe cette duplicité magistrale :

Je ne puis m'empêcher de penser qu'il y a du système et du travail dans cette attitude parfaitement triste et dans cet absolu de dégoût. Une phrase bien accordée exclut la renonciation totale. (p. 797)

Paradoxalement pour un partisan du métier et de la fabrication, Paul Valéry vilipende le souci de la forme au nom d'une forme de détachement de soi. Il fustige « une détresse qui écrit bien » (p. 797) et montre du doigt « le moi haïssable» de Pascal (2009, p. 344, S. 494). Le paradoxe, c'est qu'ici, Valéry semble dénoncer tout ce que par ailleurs il revendique. Et il

11 On reprend ici les termes d'une triade essentielle dans la conception valéryenne de l'artiste : « Manière d'être, pouvoir, savoir, vouloir ». 
regrette ce qu'il rejette d'ordinaire, la coïncidence de la littérature avec la vie, l'écriture comme expression. Il y a une aporie dans ce reproche dans la mesure où le luxe, la puissance rhétorique de la littérature offusqueraient la sincérité, exigeant du même coup une écriture de type autobiographique. Valéry éprouve ici comme une impossibilité entre la résonance de la rhétorique pascalienne et le dénuement de son objet, la détresse humaine. $\mathrm{Y}$ aurait-il donc des sujets impossibles ou interdits à la littérature ?

Mais il formule un argument équivoque, « Je vois trop la main de Pascal» (p. 797), qui confond l'exigence apologétique de l'écrivain, la facilité rhétorique et la visée morale : «Qu'est-ce que l'Éloquence?»(p.804) et «qu'est-ce que nous apprenons aux autres hommes? » (p. 797)

À la question de l'insincérité de Pascal, Maurice Blanchot, dans La Part du feu, apporte une très profonde réponse : "Le langage des Pensées, pour être vrai, doit être un langage comblé par l'existence. [...] En réalité, ce que postulent, ou ce qu'exigent Les Pensées, ce ne sont pas les détails de sa vie, ou les détails de sa sincérité, mais l'existence dans son entier [...] l'existence comme telle. » (2009, p. 256-257) À quoi répond implicitement ce propos de Valéry, qui témoigne d'un malaise sourd, d'une angoisse du pathos:

Il y a aussi je ne sais quoi de trouble, et je ne sais quoi de facile, dans la spécialité que l'on se fait des motifs tragiques et des objets impressionnants. Qu'est-ce que nous apprenons aux autres hommes en leur répétant qu'ils ne sont rien, que la vie est vaine, la nature ennemie, la connaissance illusoire? À quoi sert d'assommer ce néant qu'ils sont, ou de leur redire ce qu'ils savent? (p. 797) 
Néanmoins, cette étrange réflexion de critique moraliste contre Pascal, si étrangère à son auteur, nous interroge : qu'estce donc que la littérature? Une amplification, une dramatisation du réel, un malentendu, « le mal de prendre une hypallage pour une découverte, une métaphore pour une démonstration, un vomissement de mots pour un torrent de connaissances capitales, et soi-même pour un oracle, ce mal naît avec nous » (2016h, p. 833). Le malaise de Valéry, « Je ne suis pas à mon aise devant ce mélange de l'art avec la nature» (p. 797), ne tient-il pas à une conception de la littérature qui s'attache exclusivement à la poésie et laisse incertain le statut valéryen de la prose (Jarrety, 1992)? Ceci justifierait de sonder en ce sens le mélange, « le magma de mots morts, de peaux d'idée, de graisse de rhétorique» (Gide-Valéry, 1955, p. 159) pour un écrivain du pur et de l'impur.

Valéry a donc développé une réflexion théorique sur la création artistique comme l'effort de l'esprit pour conformer un vouloir à un pouvoir, et " une théorie de l'Instrument » (1997, p. 48) inscrite dans une conception de l'exercice (2016h, p. 828829), qui rapporte l'œuvre à ses possibles variations. Si l'œuvre est nécessaire, c'est d'exister en sa forme mais elle est comme une insignifiante variation car, précise Valéry, "l'écrivain rature, reprend, jusqu'à rejoindre "l'état posthume" » (2016a, p. 530). C'est dire que, par nature inachevée, l'œuvre est exercice répété de l'esprit. Mais, en outre, la littérature, selon Valéry, n'est pas «tant comme une histoire des auteurs et des accidents de leur carrière ou de leurs ouvrages, que comme une Histoire de l'esprit en tant qu'il produit ou consomme de la "littérature", et cette histoire pourrait même se faire sans que le nom d'un écrivain y fût prononcé » (2016j, p. 946). Ainsi, l'œuvre littéraire, plus encore, la prose de Paul Valéry, dans son 
mouvement inéluctable de réflexion critique, est-elle toujours à la frontière des genres, une littérature des limites. Ce formaliste, qui affirme " la condition verbale de la littérature » (2016f, p. 277) et, en tout art, la prééminence de sa matière, expose la littérature à l'exigence de l'illimité, au risque de l'impossible, et son exercice à en être l'essentielle virtualité. En ce sens, l'œuvre de Valéry est diverse jusqu'à la disparate et tend à sa perpétuelle dissémination.

On peut lire, dans le Journal de Gide, 13 février 1928 : "Préface à Lucien Leuwen, de Valéry - dans la très belle édition Schiffrin que je reçus hier soir. J'y sens, pour la première fois chez Valéry, certain désir de conciliation, certaine crainte de déplaire. Valéry oserait-il écrire aujourd'hui ses admirables pages sur Pascal de la Revue Hebdomadaire? » (p. 871)

«Bizarre Instinct» (2016e, p. 1330) et préméditation dessinent l'expérience critique dans cette Variation. Par son audace, la critique de Valéry est celle d'un auteur de la variété comme configuration de l'instabilité essentielle de toute pensée dans l'élan mimétique de l'émulation. De l'imitation des Anciens jusqu'à ce jour, en lisant en écrivant (Gracq, 1995), ainsi va toute littérature. Le nom de Pascal circule dans l'œuvre de Paul Valéry comme la forme prochaine d'une arrière-pensée irrésistible, géométrie et finesse, le moi haïssable, le silence éternel... une rumeur, la phrase de Pascal : « Elle le frappe. [...] Il l'oublie. Elle ne s'oublie pas. Elle se perpétue et se régénère en lui sans qu'il le sache. Elle travaille. » (2016g, p. 625)

Alors que l'article sur Pascal accouche d'une critique sourdement élaborée et fortement personnelle, dont les annotations résistent à de probables contestations, l'auteur de 
Note et Digression, texte d'introduction à La méthode de Léonard de Vinci, écrit :

Il n'est pas de tentation plus cuisante, ni plus intime, ni plus féconde peut-être, que celle du reniement de soi-même [...].

Relire donc, relire après l'oubli, - se relire, sans ombre de tendresse, sans paternité; avec froideur et acuité critique, et dans une attente terriblement créatrice de ridicule et de mépris, l'air étranger, l'œil destructeur, - c'est refaire ou pressentir que l'on referait, bien différemment son travail. (p. 812)

\section{Bibliographie}

ADORNO, Théodor W. (1999 [1958]), «Les écarts de Valéry », dans Notes sur la littérature, traduit de l'allemand par Sibylle Muller, Paris, Flammarion, coll. "Champs/Flammarion", p. 101-139.

BAYET, Jean. (1965), Littérature latine, Paris, Armand Colin.

BLANCHOT, Maurice. (2009 [1949]), La Part du feu, Paris, Gallimard.

CLAUDEL, Paul. (1963 [1993]), Réflexions sur la poésie, Paris, Gallimard, coll. « Folio/essais ».

GIDE, André, (1951), Journal (1889-1939), Paris, Gallimard, coll. « Bibliothèque de la Pléiade ».

GRACQ, Julien, (1995 [1980]), "En lisant en écrivant », dans OEuvres complètes, t. II, Paris, Gallimard, « Bibliothèque de la Pléiade ».

JARRETY, Michel. (1992), Paul Valéry, Paris, Hachette Supérieur, coll. « Portraits littéraires ».

LẺAUTAUD, Paul. (2005 [1968]), Journal littéraire, Paris, Mercure de France. 
MACROBE, Commentaire du Songe de Scipion, traduction de M. Nisard, Paris, Firmin-Didot, M DCCC LXXV, $<$ http://remacle.org/bloodwolf/erudits/macrobe/scipion1.htm>.

PASCAL, Blaise. (2009 [1670], [1991], [2000]), Pensées, Paris, Le Livre de Poche, coll. «Les Classiques de Poche».

VALẺRY, Paul. (1955), André Gide - Paul Valéry, Correspondance 1890-1942, préface et notes de Robert Mallet, Paris, Gallimard.

—. (1990a), Cahiers, 1894-1914, t. III, Paris, Gallimard.

—. (1990b), Cahiers, 1894-1914, t. VIII, Paris, Gallimard.

—. (1997 [1952]), Lettres à quelques-uns, Paris, Gallimard, coll. « L'Imaginaire ».

—. (1957), CEuvres, t. I, Paris, Gallimard, «Bibliothèque de la Pléiade ».

—. (2016 [1957]), CEuvres, édition, présentation et notes de Michel Jarrety, Paris, Le Livre de Poche, coll. «La Pochothèque $», 3$ vol.

—. (2016a [1938]), Degas Danse Dessin, dans éd. M. Jarrety, t. II.

—. (2016b [1896], [1926], [1946]), «Quelques pensées de Monsieur Teste », dans La Soirée avec Monsieur Teste, Monsieur Teste, dans éd. M. Jarrety, t. II.

—. (2016c [1932]), L'Idée fixe ou Deux hommes à la mer, dans éd. M. Jarrety, t. II.

—. (2016d [1939]), "Des couleurs », dans Mélange, Instants, dans éd. M. Jarrety, t. III.

—. (2016e [1931]), "Autour de Corot », dans Pièces sur l'art, dans éd. M. Jarrety, t. I.

—. (2016f [1941]), Tel Quel I, dans éd. M. Jarrety, t. III.

—. (2016g [1943]), Tel Quel II, dans éd. M. Jarrety, t. III. 
-. (2016h [1924]), Variété, Introduction à la méthode de Léonard de Vinci (1938), Note et digression (1919), dans éd. M. Jarrety, t. 1.

—. (2016i [1924]), "Variation sur une "pensée"», dans Variété, dans éd. M. Jarrety, t. I.

—. (2016j [1944]), Variété V, dans éd. M. Jarrety, t. III.

\section{Résumé}

Étudier Variation sur une "pensée» de Paul Valéry met en évidence une conception inaugurale de la littérature, pensée comme inachèvement dans la mesure où elle est le mouvement infini de l'Esprit qui rature sans fin ses œuvres. Des notes marginales glosent ainsi la Pensée de Pascal, que Valéry expose à la confrontation parodique des discours antiques. Car l'instabilité essentielle de la conscience qui pense induit nécessairement le commentaire et constitue dès lors chaque œuvre en fragment et la littérature en palimpseste. Ainsi écrire s'inscrit dans une dynamique dialogique aussi envahissante qu'heuristique.

\section{Abstract}

Studying Paul Valéry's Variation on a "thought" highlights his inaugural insight on literature, seen as a process, always unachieved for being the endless movement of the Mind scribbling its work. Thus marginal notes commenting a Thought (Pensée) of Pascal Valéry exposes to the confronting parody of ancient prose. From the essential imbalance of a consciousness thinking, needs to follow the commentary and therefore each work as fragments, literature as palimpsest. Writing enters though a dialogic dynamic as invasive as heuristic. 